Pacific

Journal of

Mathematics

\title{
THE SCHRÖDINGER EQUATION ON SPHERES
}

MichaEl TAYLOR 


\title{
THE SCHRÖDINGER EQUATION ON SPHERES
}

\author{
Michael TAYLOR
}

\begin{abstract}
It is shown that the fundamental solution to the Schrödinger equation on a $d$-dimensional sphere has an explicit description at times that are rational multiples of $\pi$. This leads to sharp $L^{p}$ estimates on the solution operator at those times. Analogous, though less explicit, results are obtained when spheres are replaced by Zoll manifolds, and when potentials are added.
\end{abstract}

\section{Introduction.}

Let $\Delta$ denote the Laplace-Beltrami operator on the $d$-dimensional sphere $S^{d}$, with its standard metric. The fundamental solution to the Schrödinger equation:

$$
i \frac{\partial u}{\partial t}=\Delta u, \quad u(0, x)=\delta_{p}(x),
$$

is a distribution on $\mathbb{R} \times S^{d}$ with fairly nasty behavior; its singular support is all of $\mathbb{R} \times S^{d}$. However, J. Rauch pointed out to me that when $d=1$ and $t$ is a rational multiple of $\pi$ (we say $t \in \pi \mathbb{Q}$ ), then $e^{-i t \Delta} \delta(x) \in \mathcal{D}^{\prime}\left(S^{1}\right.$ ) is a finite sum of delta functions on $S^{1}$. Hence for such $t \in \pi \mathbb{Q}, e^{-i t \Delta}$ is bounded on $L^{p}\left(S^{1}\right)$ for each $p \in[1, \infty]$. Here we work out an equally precise description of $e^{-i t \Delta}$ on $\mathcal{D}^{\prime}\left(S^{d}\right)$, for each $t \in \pi \mathbb{Q}$. From this follows a precise account of the $L^{p}$-Sobolev mapping properties of $e^{-i t \Delta}$, for such $t$.

In $\S 2$ we will derive the basic identities for $e^{-i t \Delta}$ on $\mathcal{D}^{\prime}\left(S^{d}\right)$ when $t \in \pi \mathbb{Q}$. For such $t$ we express $e^{-i t \Delta}$ in terms of solution operators to a wave equation. This leads to the sharp $L^{p}$-Sobolev estimates. Establishing sharpness is simply a matter of showing that certain coefficients in the formula for $e^{-i t \Delta}$ do not vanish. This issue is settled in $\S 3$.

In $\S 4$ we discuss various extensions of these results. It is mentioned that another extension of the $S^{1}$ case is to $d$-dimensional tori, and that formulas there have a number-theoretical significance. We also discuss extensions to Zoll surfaces and to situations where a potential is added to the Laplace operator. 


\section{Basic identities.}

For $d=1$, we have the Fourier series representation for $S(t, x)=e^{-i t \Delta} \delta(x)$ at $t=2 \pi m / n$ :

$$
S(2 \pi m / n, x)=\frac{1}{2 \pi} \sum_{\nu=-\infty}^{\infty} e^{2 \pi i \nu^{2} m / n} e^{i \nu x} .
$$

If we set $\nu=n j+\ell$ and produce a double sum over $j \in \mathbb{Z}, \ell \in\{0, \ldots, n-1\}$, the sum over $j$ becomes

$$
\sum_{j=-\infty}^{\infty} e^{i n j x}=\frac{2 \pi}{n} \sum_{j=0}^{n-1} \delta_{2 \pi j / n}
$$

and we obtain the distribution on $S^{1}=\mathbb{R} /(2 \pi \mathbb{Z})$ :

$$
S(2 \pi m / n, x)=\frac{1}{n} \sum_{j=0}^{n-1} G(m, n, j) \delta_{2 \pi j / n}(x),
$$

with

$$
G(m, n, j)=\sum_{\ell=0}^{n-1} e^{2 \pi i\left(\ell^{2} m+\ell j\right) / n} .
$$

Let us note that the sum is really over $\ell \in \mathbb{Z} /(n)$. In particular we can replace $\ell$ by $-\ell$ and hence see that $G(m, n, j)$ is even in $j$. We also note that our formulas implicitly assume $n>0$, but we need make no restriction on the sign of $m$.

The following alternative presentation of (2.3) has some advantages: Set

$$
\Gamma(m, k, j)=\frac{1}{2 k} G(m, 2 k, j)=\frac{1}{2 \pi} \sum_{\ell=0}^{2 k-1} e^{\pi i\left(\ell^{2} m+\ell j\right) / k} .
$$

If we set $n=2 k$ in $(2.3)$, we have

$$
S(\pi m / k, x)=\sum_{j=0}^{2 k-1} \Gamma(m, k, j) \delta_{\pi j / k}(x) .
$$

These formulas will prove useful in our analysis of the higher-dimensional case.

We now consider $e^{-i t \Delta}$ on $S^{d}$. As is common in analysis on spheres, we take

$$
A=\sqrt{-\Delta+\left(\frac{d-1}{2}\right)^{2}}
$$


It is well-known that

$$
\operatorname{Spec} A=\left\{\frac{d-1}{2}+j: j=0,1,2, \ldots\right\} .
$$

In particular $\operatorname{Spec} A \subset \mathbb{Z}$ if $d$ is odd and $\operatorname{Spec} A \subset \mathbb{Z}+\frac{1}{2}$ if $d$ is even.

Now when $\operatorname{Spec} A \subset \mathbb{Z}$ we can analyze functions of the self-adjoint operator $A$ by the formula

$$
\varphi(A)=\int_{S^{1}} \hat{\varphi}(t) \cos t A d t,
$$

provided $\varphi$ is even, with

$$
\hat{\varphi}(t)=\frac{1}{2 \pi} \sum_{\nu=-\infty}^{\infty} \varphi(\nu) e^{i \nu t} .
$$

This is easily confirmed via the spectral theorem and Fourier inversion, if $\varphi: \mathbb{Z} \rightarrow \mathbb{C}$ is summable. Then a limiting argument gives it for any bounded (or even polynomially bounded) $\varphi$ (yielding $\hat{\varphi} \in \mathcal{D}^{\prime}\left(S^{1}\right)$ ), exploiting the smooth dependence on $t$ of the family of operators $\cos t A$, acting on $\mathcal{D}^{\prime}\left(S^{d}\right)$. In particular we can apply $(2.9)$ with $\varphi(\nu)=\varphi_{s}(\nu)=e^{i s \nu^{2}}$. If $s=m \pi / k$, then $\hat{\varphi}_{s}$ is given by $(2.1)$, with $n=2 k$, so by (2.6) we have

$$
e^{\pi i(m / k) A^{2}}=\sum_{j=0}^{2 k-1} \Gamma(m, k, j) \cos \frac{\pi j}{k} A,
$$

yielding a formula for

$$
e^{-i t \Delta}=e^{-i t(d-1)^{2} / 4} e^{i t A^{2}}
$$

at $t=m \pi / k$, on $\mathcal{D}^{\prime}\left(S^{d}\right)$, when $d$ is odd.

In case $\operatorname{Spec} A \subset \mathbb{Z}+\frac{1}{2}$, then $\operatorname{Spec} 2 A \subset \mathbb{Z}$, and (2.9) applies with $A$ replaced by $2 A$. Taking $\varphi(\nu)=\varphi_{s}(\nu)$ as before, we have

$$
e^{4 \pi i(m / k) A^{2}}=\sum_{j=0}^{2 k-1} \Gamma(m, k, j) \cos \frac{2 \pi j}{k} A,
$$

yielding a formula for $e^{-i t \Delta}$ at $t=4 \pi m / k$ on $\mathcal{D}^{\prime}\left(S^{d}\right)$, valid for $d$ even (and also for $d$ odd).

Note that $\cos t A$ is a solution operator for a hyperbolic PDE. In fact, given $f \in \mathcal{D}^{\prime}\left(S^{d}\right), u(t, x)=(\cos t A) f(x)$ solves

$$
u_{t t}-\left(\Delta-\left(\frac{d-1}{2}\right)^{2}\right) u=0, \quad u(0, x)=f(x), u_{t}(0, x)=0 .
$$

In particular, for each $t, \cos t A$ is a Fourier integral operator of order zero. Its mapping properties on $L^{p}$ Sobolev spaces $H^{s, p}$ are well-known (cf. [S3]), and by (2.11) and (2.13) they are shared by $e^{-i t \Delta}$ for each $t \in \pi \mathbb{Q}$. Thus we have the following: 
Proposition 2.1. Given $p \in(1, \infty), s \in \mathbb{R}$, we have

$$
e^{-\pi i(m / k) \Delta}: H^{s, p}\left(S^{d}\right) \longrightarrow H^{s-(d-1)|1 / 2-1 / p|, p}\left(S^{d}\right) .
$$

Such estimates also hold in the endpoint cases $p=1, \infty$, with $L^{1}$ replaced by the local Hardy space $\mathfrak{h}^{1}$ and $L^{\infty}$ replaced by bmo.

It is well-known that such a mapping property cannot be improved for $\cos t A$ when $0<t<\pi$. We aim to show that (2.15) cannot be improved, with some obvious exceptions, noted in $\S 3$. In view of (2.11) and (2.13) this merely amounts to examining whether cancellations can arise. In fact (2.11) and (2.13) can be collapsed somewhat, to involve $\cos t_{\nu} A$ with $\left\{t_{\nu}\right\}$ running over $[0, \pi]$, without multiplicities. We take up the task of doing this.

\section{Further analysis of the coefficients.}

First look at (2.11), and note that $\Gamma(m, k, j)$, given by $(2.5)$, is even in $j$ and periodic of period $2 k$ in $j$, so $\Gamma(m, k, j)=\Gamma(m, k, 2 k-j)$. Also, when $\operatorname{Spec} A \subset \mathbb{Z}, \cos (2 \pi-t) A=\cos t A$, so $\cos (\pi j / k) A$ is unchanged when $j$ is replaced by $2 k-j$. Thus we have on $\mathcal{D}^{\prime}\left(S^{d}\right)$ for $d$ odd:

$$
e^{\pi i(m / k) A^{2}}=\Gamma(m, k, 0) I+\Gamma(m, k, k) P+2 \sum_{j=1}^{k-1} \Gamma(m, k, j) \cos \frac{\pi j}{k} A .
$$

Here $I$ is the identity operator and $P=\cos \pi A$. When $A$ is given by (2.7) on $\mathcal{D}^{\prime}\left(S^{d}\right)$, with $d$ odd, we have

$$
\operatorname{Pf}(x)=(-1)^{(d-1) / 2} f(-x) .
$$

Looking at (2.13), we also see that

$$
e^{4 \pi i(m / k) A^{2}}=\Gamma(m, k, 0) I+\Gamma(m, k, k) Q+2 \sum_{j=1}^{k-1} \Gamma(m, k, j) \cos \frac{2 \pi j}{k} A .
$$

In this case $Q=\cos 2 \pi A$. From (2.8) we see that $Q=(-1)^{d-1} I$. In other words,

$$
\begin{aligned}
e^{4 \pi i(m / k) A^{2}}= & \left\{\Gamma(m, k, 0)+(-1)^{d-1} \Gamma(m, k, k)\right\} I \\
& +2 \sum_{j=1}^{k-1} \Gamma(m, k, j) \cos \frac{2 \pi j}{k} A .
\end{aligned}
$$

Now we have terms $\cos t_{j} A$, with $t_{j} \in(0,2 \pi)$, and we still want to cut the sum down. This time, use

$$
\cos (2 \pi-t) A=(\cos 2 \pi A) \cos t A-(\sin 2 \pi A) \sin t A .
$$

As seen above, $\cos 2 \pi A=(-1)^{d-1} I$, and meanwhile Spec $2 A \subset \mathbb{Z} \Rightarrow \sin 2 \pi A$ $=0$, so we have $\cos (2 \pi-t) A=(-1)^{d-1} \cos t A=-\cos t A$ if $d$ is even, which 
we assume from here to the end of formula (3.8) below. To work on the sum over $j$ in (3.4), we consider separately two cases.

First suppose $k=2 \nu$ is even. Then we can write the sum over $1 \leq j \leq$ $k-1$ in $(3.4)$ as

$$
\sum_{j=1}^{k / 2-1}\{\Gamma(m, k, j)-\Gamma(m, k, k-j)\} \cos \frac{2 \pi j}{k} A+\Gamma(m, k, \nu) \cos \pi A .
$$

Recalling that $\operatorname{Spec} A \subset \mathbb{Z}+\frac{1}{2}$ for $d$ even, we have $\cos \pi A=0$, so in this case we have

$$
\begin{aligned}
e^{4 \pi i(m / k) A^{2}}= & \{\Gamma(m, k, 0)-\Gamma(m, k, k)\} I \\
& +2 \sum_{j=1}^{k / 2-1}\{\Gamma(m, k, j)-\Gamma(m, k, k-j)\} \cos \frac{2 \pi j}{k} A .
\end{aligned}
$$

Next suppose $k=2 \nu+1$ is odd. Then we have

$$
\begin{aligned}
e^{4 \pi i(m / k) A^{2}}= & \{\Gamma(m, k, 0)-\Gamma(m, k, k)\} I \\
& +2 \sum_{j=1}^{(k-1) / 2}\{\Gamma(m, k, j)-\Gamma(m, k, k-j)\} \cos \frac{2 \pi j}{k} A .
\end{aligned}
$$

To reiterate, (3.7) and (3.8) hold on $\mathcal{D}^{\prime}\left(S^{d}\right)$ for $d$ even.

Now at this point there are no cancellations between terms in any of the formulas (3.1), (3.7), and (3.8). To be precise, suppose $f$ is supported in a ball of radius $<1 / 2 k$ in $S^{d}$. Then, in each of these formulas, we have that the various terms $\left(\cos t_{\nu} A\right) f$ that arise have disjoint singular support. There remains the issue of whether the coefficients of these various terms $\cos t_{\nu} A$ might vanish. In fact, some do, and we now take up the question of exactly which coefficients vanish and which do not.

We mention some properties of $\Gamma(m, k, j)$, whose proofs are given in [HB], and also in [T2]. First, $\Gamma(m, k, j)$ can vanish sometimes. In fact

$$
m k+j \text { odd } \Longrightarrow \Gamma(m, k, j)=0 .
$$

Now let us set $\Gamma(m, k)=\Gamma(m, k, 0)$. There is the following result of $[\mathbf{H B}]$ :

Lemma 3.1. Assume that $m$ and $k$ are relatively prime.

(i) If $m k$ and $j$ are even, then, with $\mu$ solving $\mu m=1 \bmod k$,

$$
\Gamma(m, k, j)=e^{-\pi i(m / k)(j / 2)^{2} \mu^{2}} \Gamma(m, k) .
$$

(ii) If $m k$ and $j$ are odd, then, with $\nu$ solving $4 \nu m=1 \bmod k$,

$$
\Gamma(m, k, j)=e^{-4 \pi i(m / k) \nu^{2} j^{2}} \Gamma(4 m, k) .
$$


Furthermore, in Cases (i) and (ii), respectively, we have

$$
|\Gamma(m, k)|=k^{-1 / 2}, \quad|\Gamma(4 m, k)|=k^{-1 / 2} .
$$

In particular, when $m$ and $k$ are relatively prime,

$$
m k+j \text { even } \Longrightarrow \Gamma(m, k, j) \neq 0 .
$$

The results (3.9) and (3.13) specify precisely which coefficients arising in (3.1), for $e^{\pi i(m / k) A^{2}}$ on $\mathcal{D}^{\prime}\left(S^{d}\right)$ with $d$ odd, are nonvanishing, when $m$ and $k$ are relatively prime. It remains to look at the coefficients that arise in (3.7)-(3.8), describing $e^{4 \pi i(m / k) A^{2}}$ on $\mathcal{D}^{\prime}\left(S^{d}\right)$ for $d$ even. Again we take $m$ and $k$ to be relatively prime. We consider three main cases, each having two subcases.

Case (I): $m$ even, $k$ odd.

$$
\begin{aligned}
j \text { even } & \Longrightarrow \Gamma(m, k, j) \neq 0 \text { and } \Gamma(m, k, k-j)=0, \\
j \text { odd } & \Longrightarrow \Gamma(m, k, j)=0 \text { and } \Gamma(m, k, k-j) \neq 0 .
\end{aligned}
$$

In both subcases, $\Gamma(m, k, j)-\Gamma(m, k, k-j) \neq 0$.

Case (II): $m$ odd, $k$ even.

$$
\begin{aligned}
& j \text { odd } \Longrightarrow \Gamma(m, k, j)=\Gamma(m, k, k-j)=0, \\
& j \text { even } \Longrightarrow \Gamma(m, k, j)-\Gamma(m, k, k-j) \\
& \quad=\left\{e^{-\pi i(m / k)(j / 2)^{2} \mu^{2}}-e^{-\pi i(m / k)(k / 2-j / 2)^{2} \mu^{2}}\right\} \Gamma(m, k) .
\end{aligned}
$$

We take a closer look at this expression. Recall that $|\Gamma(m, k)|=k^{-1 / 2}$ in this case. Meanwhile the quantity in braces is equal to

$$
e^{-\pi i(m / k)(j / 2)^{2} \mu^{2}}\left[1-e^{-\pi i(m / k)\left(k^{2} / 4-j k / 2\right) \mu^{2}}\right] .
$$

We look at the exponent in the last exponential in (3.14). Say $\mu m=1+$ $a k, a \in \mathbb{Z}$. Then $(\mu+b k) m=1+(a+b m) k=1+a_{1} k$, and since $m$ is odd we can arrange that $a_{1}$ be even. The quantity (3.10) is independent of the choice of $\mu \bmod k$, so we can just assume $a$ is even. Now the exponent mentioned above is seen to be the negative of

$$
\pi i \mu\left(\frac{k}{4}-\frac{j}{2}\right)+\pi i a \mu\left(\frac{k^{2}}{4}-\frac{j k}{2}\right),
$$

so the quantity (3.14) vanishes if and only if $k / 4-j / 2$ is an even integer.

Case (III): $m$ odd, $k$ odd.

$$
\begin{array}{r}
j \text { even } \Longrightarrow \Gamma(m, k, j)=0 \text { and } \Gamma(m, k, k-j) \neq 0, \\
j \text { odd } \Longrightarrow \Gamma(m, k, j) \neq 0 \text { and } \Gamma(m, k, k-j)=0 .
\end{array}
$$


These considerations specify all the coefficients arising in (3.1), (3.7), and (3.8), except that (3.12) specifies $\Gamma(m, k)$ (for $m k$ even) only up to phase. Further specification can be found in [T2].

Having these formulas for $e^{-i t \Delta}$ when $t \in \pi \mathbb{Q}$, we note that there are explicit formulas for the action of $\cos t A$ on functions on $S^{d}$. We write them down here (at least for $d$ odd). Demonstrations can be found in Chapter 8 of [T1]. If $d=2 \nu+1$ is odd, we have

$$
(\cos t A) f(x)=C_{\nu}(\sin t)\left(\frac{1}{\sin t} \frac{\partial}{\partial t}\right)^{\nu}\left(\sin ^{2 \nu-1} t \bar{f}_{x}(t)\right),
$$

for $0<t<\pi$, where $\bar{f}_{x}(t)$ is the mean value of $f$ over the shell

$$
\Sigma_{x}(t)=\left\{y \in S^{d}: \operatorname{dist}(x, y)=t\right\},
$$

and where $\operatorname{dist}(x, y)$ denotes the spherical distance. Here $C_{\nu}=1 /(2 \nu-1) !$ !, where $(2 \nu-1) ! !=3 \cdot 5 \ldots(2 \nu-1)$. Note that the strong Huygens principle holds here; $(\cos t A) \delta_{p}$ is supported on the shell $\Sigma_{p}(t)$. If $d=2 \nu$ is even, there is a formula in similar analogy to the solution to the wave equation on $\mathbb{R} \times \mathbb{R}^{d}$ for $d$ even; we refer to [T1] for details. Of course, in this case the strong Huygens principle fails, but the singular support of $(\cos t A) \delta_{p}$ lies on $\Sigma_{p}(t)$.

We apply the results of this section to demonstrate the sharpness of the operator regularity stated in (2.15), with a discrete set of exceptions. Some of the exceptions are apparent from the fact that $\operatorname{Spec}(-\Delta)=\{j(j+d-1)$ : $j=0,1,2, \ldots\}$, hence consists of integers (even integers if $d$ is even). Thus $e^{-\pi i t \Delta}=I$ on functions on $S^{d}$ whenever $d$ is odd and $t$ is an even integer, and whenever $d$ is even and $t$ is an integer. We check this observation against the formulas (3.1) and (3.7)-(3.8). Doing so will produce another discrete set of $t$ s for which $e^{-\pi i t \Delta}$ is bounded on all the spaces $H^{s, p}\left(S^{d}\right)$ and show that for all other $t \in \mathbb{Q}$ the operator mapping properties given in (2.15) cannot be improved.

First suppose $d$ is odd, so (3.1) applies. Assume $m$ and $k$ are relatively prime. The "wave contribution" is

$$
2 \sum_{j=1}^{k-1} \Gamma(m, k, j) \cos \frac{\pi j}{k} A .
$$

If $k=1$, this sum is empty, and we have $e^{\pi i m A^{2}}=\Gamma(m, 1,0) I+\Gamma(m, 1,1) P$. When $m$ is even, $\Gamma(m, 1,1)=0$ and we recover the observation made in the previous paragraph. If $k=2$ in (3.1), then the sum (3.17) has one term, involving $\Gamma(m, 2,1)$, which vanishes, by $(3.9)$, so $e^{\pi i(m / 2) A^{2}}=\Gamma(m, 2,0) I+$ $\Gamma(m, 2,2) P$. Hence $e^{-\pi i t \Delta}: H^{s, p}\left(S^{d}\right) \rightarrow H^{s, p}\left(S^{d}\right)$ for all $s, p$ if $d$ is odd and $2 t \in \mathbb{Z}$. If $k \geq 3$ in (3.1), then the sum (3.17) contains terms involving the coeffcients $\Gamma(m, k, 1)$ and $\Gamma(m, k, 2)$, and by (3.13) at least one of these terms is not zero, so (2.15) cannot be improved. 
Next suppose $d$ is even, so (3.7) applies to $e^{-\pi i t \Delta}$ if $t=4 m / k$ with $k$ even, and (3.8) applies if $k$ is odd. Assume $m$ and $k$ are relatively prime. This time the "wave contribution" is

$$
2 \sum_{j=1}^{[(k-1) / 2]}\{\Gamma(m, k, j)-\Gamma(m, k, k-j)\} \cos \frac{2 \pi j}{k} A .
$$

If $k=1$ or 2 , this sum is empty. If $k=4$, the sum (3.18) has one term, with coefficient $\Gamma(m, 4,1)-\Gamma(m, 4,3)=0$. These results recover the observation that $e^{-\pi i t \Delta}=I$ for $t \in \mathbb{Z}$. If $k \geq 3$ is odd, then Cases (I) and (III) show none of the terms in (3.18) vanish. If $k \geq 6$ is even, a check of Case (II) shows some of the terms in (3.18) can vanish, but in no cases do all of them vanish, so there are no further cases when $(2.15)$ can be improved. In particular, for $k=6$ one has $\Gamma(m, 6,2)-\Gamma(m, 6,4) \neq 0$ and for $k=8$ one has $\Gamma(m, 8,2)-\Gamma(m, 8,6) \neq 0$.

\section{Remarks and extensions.}

Here we make several remarks on applications and extensions of the calculations made in $\S 2$.

Remark 1. The quantities $G(m, n, j)$ and $\Gamma(m, k, j)$ defined in (2.4)-(2.5), which arise in the calculation of $S(2 \pi m / n, x)$, are Gauss sums, of interest in number theory. Another way to evaluate $S(2 \pi m / n, x)$ is to take the free-space fundamental solution $S_{0}(t, x)$ giving $e^{-i t \Delta}$ on $\mathbb{R} \times \mathbb{R}$ and sum its translates $S_{0}(t, x-2 \pi \nu), \nu \in \mathbb{Z}$. Carrying this out yields a formula similar to (2.3), but with coefficients involving different Gauss sums. Comparing the calculations produces a straightforward and nifty proof of the reciprocity formula for Gauss sums:

$$
\sum_{\ell=0}^{n-1} e^{2 \pi i \ell^{2} m / n} e^{2 \pi i \ell j / n}=\frac{1+i}{2}\left(\frac{n}{m}\right)^{1 / 2} e^{-\pi i j^{2} / 2 m n} \sum_{\ell=0}^{2 m-1} e^{-\pi i \ell^{2} n / 2 m} e^{\pi i j \ell / m} .
$$

This formula was first derived (by different means) by Landsberg and Schaar, in the 1890s. Specializing to $j=0, m=1$ gives the classical formula

$$
\sum_{\ell=0}^{n-1} e^{2 \pi i \ell^{2} / n}=\frac{1+i}{2}\left(1+i^{-n}\right) \sqrt{n}
$$

due to Gauss, used in one of his proofs of the quadratic reciprocity formula. 
Remark 2. Another multidimensional extension of (2.3) involves $e^{i t Q(D)} \delta(x)$ on the torus $\mathbb{T}^{d}=\mathbb{R}^{d} /(2 \pi \mathbb{Z})^{d}$, where

$$
Q(\xi)=\xi \cdot A \xi, \quad A^{t}=A \in G \ell(d, \mathbb{Z}), \quad \operatorname{det} A= \pm 1 .
$$

Then $e^{i t Q(D)} \delta(x)$ can be evaluated for $t \in \pi \mathbb{Q}$. Again two methods work. One involves Fourier series on $\mathbb{T}^{d}$. The other involves writing down the fundamental solution $S_{0}^{Q}(t, x)$ giving $e^{i t Q(D)}$ on $\mathbb{R} \times \mathbb{R}^{d}$ and summing $S_{0}^{Q}(t, x+$ $2 \pi \nu)$ over $\nu \in \mathbb{Z}^{d}$. Both produce finite linear combinations of delta functions supported on a lattice in $\mathbb{T}^{d}$, and comparing calculations produces reciprocity formulas for multivariate Gauss sums, obtained (by different means) in $[\mathbf{K}]$. The reader can try this as an exercise, or see $[\mathbf{T 2}]$ for details.

Remark 3. There are other extensions of the material presented above, arising from the fact that the identity (2.11) is valid whenever $A$ is a selfadjoint operator with spectrum contained in $\mathbb{Z}$. This can be applied as follows: Let $M$ be a $d$-dimensional Zoll manifold, a compact Riemannian manifold on which all geodesics have minimal period $2 \pi$, and consider $H=$ $-\Delta+V$, with positive, real-valued $V \in C^{\infty}(M)$. As shown in $[\mathbf{C d V}]$ (cf. also $\S 29.2$ of $[\mathbf{H}]$ ), there exists a positive, self-adjoint $A \in O P S^{1}(M)$ and $S \in O P S^{-1}$, commuting with each other (and with $H$ ) and $\alpha \in \mathbb{R}$, such that

$$
\sqrt{-\Delta+V}=A+\alpha I+S, \quad \text { Spec } A \subset \mathbb{Z} .
$$

(Here $O P S^{m}(M)$ denotes the space of $m$ th order pseudodifferential operators of classical type on $M$.) Then

$$
e^{i t(-\Delta+V)}=e^{i t\left(\alpha^{2}+2 A S+2 \alpha S+S^{2}\right)} e^{2 i \alpha t A} e^{i t A^{2}} .
$$

Noting that $e^{i t A}$ is a group of operators with the same Sobolev space mapping properties as used to prove Proposition 2.1, and that the first factor on the right side of $(4.5)$ is a family of operators in $O P S^{0}(M)$, and applying (2.11) to $e^{i t A^{2}}$ for $t \in \pi \mathbb{Q}$, we see that

$$
e^{\pi i(m / k)(-\Delta+V)}: H^{s, p}(M) \longrightarrow H^{s-(d-1)|1 / 2-1 / p|, p}(M),
$$

extending (2.15) to this context.

Remark 4. In [GGR] it is shown that, when $d=1, e^{-i t \Delta}$ does not map $L^{p}\left(S^{1}\right)$ to itself for any $p \neq 2$ when $t \notin \pi \mathbb{Q}$. By contrast with such an indication that $S(t, \cdot) \in \mathcal{D}^{\prime}\left(S^{1}\right)$ is less regular for $t \notin \pi \mathbb{Q}$ than for $t \in \pi \mathbb{Q}$, results in $[\mathbf{K R}]$ give a sense in which $S(t, \cdot) \in \mathcal{D}^{\prime}\left(S^{1}\right)$ is more regular for (most) $t \notin \pi \mathbb{Q}$ than for $t \in \pi \mathbb{Q}$. Here regularity is measured in the scale of Besov spaces $B_{\infty, \infty}^{s}\left(S^{1}\right)$. It is clear from Formula $(2.3)$ that $S(t, \cdot) \in$ $B_{\infty, \infty}^{-1}\left(S^{1}\right)$ for $t \in \pi \mathbb{Q}$ and one cannot improve this. It is shown in $[\mathbf{K R}]$ 
that, for a.e. $t \in \mathbb{R}$,

$$
S(t, \cdot) \in B_{\infty, \infty}^{-s}\left(S^{1}\right), \quad \forall s>\frac{1}{2} .
$$

More precise results relate the best $s$ to the continued fraction expansion of $t / \pi$; see $[\mathbf{K R}]$ for details.

It is tempting to speculate that $S(t, \cdot) \in \mathcal{D}^{\prime}\left(S^{1}\right)$ is more regular in $B_{p, p}^{-s}\left(S^{1}\right)$ for rational than for irrational $t / \pi$ when $p<2$ and more regular for irrational than for rational $t / \pi$ when $p>2$. But at this point this is just a speculation.

Remark 5. We also mention the recent paper [BGT], dealing with the Schrödinger equation on a compact $d$-dimensional Riemannian manifold $M$. It is shown that, given $\varphi \in C_{0}^{\infty}(\mathbb{R})$, there exists $\alpha>0$ such that, for $h \in(0,1]$,

$$
\left\|e^{-i t \Delta} \varphi\left(h^{2} \Delta\right) f\right\|_{L^{\infty}(M)} \leq C|t|^{-d / 2}\|f\|_{L^{1}(M)}, \quad|t| \leq \alpha h .
$$

This is used to produce Strichartz estimates, leading to solvability results for nonlinear Schrödinger equations. In [BGT], the sharpness of some of their Strichartz estimates is verified in particular for $M=S^{d}$, with its standard metric.

Acknowledgment. Thanks to Jeffrey Rauch for several interesting conversations related to this work. This work was performed while the author was at MSRI, and was partially supported by NSF grant DMS-9877077.

\section{References}

[BGT] N. Burq, P. Gérard and N. Tzvetkov, The Schrödinger equation on a compact manifold: Strichartz estimates and applications, Jour. 'Equations aux Derivées Partielles' (Plestin-les-Graves, 2001), Exposé No. 5, 18 pp., Univ. Nantes, 2001, MR 2003a:58055.

[CdV] Y. Colin de Verdière, Sur le spectre des opérateurs elliptiques à bicharacteristiques toutes périodiques, Comment. Math. Helv., 54 (1979), 508-522, MR 81a:58052, Zbl 0459.58014

[GGR] P. Gérard, F. Golse and J. Rauch, unpublished manuscript.

[HB] J. Hannay and M. Berry, Quantization of linear maps on a torus - Fresnel diffraction by a periodic grating, Physica, 1D (1980), 267-290, MR 82g:81005.

$[\mathrm{H}] \quad$ L. Hörmander, The Analysis of Linear Partial Differential Operators, Vol. 4, Springer-Verlag, New York, 1985, MR 98f:35002, Zbl 0612.35001.

[KR] L. Kapitanski and I. Rodnianski, Does a quantum particle know the time?, in 'Emerging Applications in Number Theory', 355-371, D. Hejhal et al. (eds.), Springer-Verlag, New York, 1999, MR 2000g:81044.

[K] A. Krazer, Zur Theorie der mehrfachen Gausschen Summen, H. Weber Fetschrift, Leipzig, 1912, 181. 
[S3] A. Seeger, C. Sogge and E. Stein, Regularity properties of Fourier integral operators, Ann. of Math., 134 (1991), 231-251, MR 92g:35252, Zbl 0754.58037.

[T1] M. Taylor, Partial Differential Equations, Vol. 2, Springer-Verlag, New York, 1996, MR 98b:35003, Zbl 0869.35003.

[T2] _ Tidbits in Harmonic Analysis, Lecture Notes, UNC, 1998.

Received December 10, 2001 and revised March 25, 2002.

Mathematics Department

University of North Carolina

Chapel Hill, NC 27599

E-mail address: met@math.unc.edu 